\title{
Políticas de inclusión digital en Argentina y el Cono sur: cartografía, perspectivas y problemáticas
}

\author{
Lago Silvia Martínez, ${ }^{1}$ \\ Gendler, Martín ${ }^{2}$ \\ Méndez, Anahiß
}

\section{Resumen}

El artículo conforma un recorte de la investigación "Políticas Públicas para la inclusión digital en Argentina y el Cono Sur"4. Se presenta un estado del arte sobre las políticas de inclusión digital que se implementan en la actualidad en Brasil, Uruguay, Chile, Perú, Ecuador y Argentina. Asimismo, se analizan los conceptos de brecha digital, inclusión y exclusión digital en el marco del análisis de la problemática a nivel regional. Por último el trabajo se focaliza en una política pública del Estado Nacional Argentino para la inclusión digital, el Programa Núcleos de Acceso al Conocimiento (NAC). Se analizan los avances producidos en la investigación sobre la base de entrevistas a coordinadores, observaciones y una encuesta, realizadas en NAC emplazados en el área metropolitana de Buenos Aires, en la Provincia de Buenos Aires y en la ciudad de Córdoba.

Inclusión digital. Brecha digital. Políticas públicas. Núcleos de Acceso al Conocimiento

\section{Resumo}

O artigo traz um estado da arte sobre políticas de inclusão digital que são implantados hoje no Brasil, Uruguai, Chile, Peru, Equador e Argentina e apresenta os conceitos de exclusão digital, a inclusão digital e da exclusão sob a análise dos problemas a nível regional são analisados. Finalmente, o trabalho centra-se na política pública do governo argentino para a inclusão digital, o Programa de Acesso conhecimento básico (PAC) trazendo os avanços na pesquisa com base em entrevistas com coordenadores, observações e uma pesquisa realizada em NAC, implantado na área metropolitana de Buenos Aires, na província de Buenos Aires e Córdoba.

Inclusão digital. Abismo digital. Políticas públicas. Núcleos de acesso ao conhecimento

\footnotetext{
${ }^{1}$ Socióloga, profesora de la Facultad de Ciencias Sociales de la UBA e investigadora del Instituto Gino Germani donde co-dirige el Programa de Investigaciones sobre la Sociedad de la Información. Es especialista en políticas científicas y tecnológicas y en su trabajo de investigación desarrolla varias líneas en los temas de tecnología y sociedad y cultura digital. slagomartinez@ gmail.com

2 Sociólogo, docente y doctorando en Ciencias Sociales en la Universidad de Buenos Aires. Becario doctoral del CONICET y miembro del Programa de investigaciones sobre la Sociedad de la Información del Instituto Gino Germani. martin.gendler@gmail.com

${ }^{3}$ Socióloga, docente y maestranda en Comunicación y Cultura de la Universidad de Buenos Aires. Becaria UBACyT y miembro del Programa de investigaciones sobre la Sociedad de la Información del Instituto Gino Germani. anahimendez.86@gmail.com
} 


\section{Introducción}

\section{Brecha, inclusión y exclusión digital}

El paradigma de la sociedad de la información y del conocimiento, ha generado a nivel mundial y en América Latina y el Caribe en particular, una diversidad de políticas públicas asociadas con el desarrollo de las naciones en la economía global. Autores como Mattelart (2002), Crovi Druetta (2004), Valderrama (2012), analizan la "Sociedad de la Información y del Conocimiento" como un proyecto hegemónico gestado por los gobiernos de los países de la OCDE, las Naciones Unidas y la Comunidad Europea durante las décadas de los ochenta y noventa. Según Valderrama, (2012, p. 14) en el año 2000, los países miembros del G-8, reunidos en Okinawa (Japón), decidieron impulsar de manera explícita el proyecto Sociedad global de la Información. Esta apuesta política y de desarrollo económico se consolidó en la Cumbre de Génova en 2001 y en las Cumbres Mundiales sobre la Sociedad de la Información (CMSI) de Ginebra 2003 y Túnez 2005. En consecuencia, los países de la región latinoamericana adoptan los principios de la CMSI y el término "Sociedad de la Información" y nutren sus agendas de políticas y estrategias ligadas al ritmo que sugieren los organismos multilaterales (Lago Martínez, 2005, p.266).

Al mismo tiempo, se impone el concepto de "brecha digital" para dar cuenta de las desigualdades tecnológicas entre los países, las regiones, las ciudades y las comunidades. Las políticas públicas en materia tecnológica que son implementadas desde los años ' 90 en la región, han tenido entre sus principales objetivos la disminución de la brecha digital.

Más recientemente se adopta la noción multidimensional de inclusión/exclusión digital íntimamente vinculada al concepto de brecha digital. Esta noción emerge con el desarrollo de la primera fase de la Cumbre Mundial de la Sociedad de la Información en el año $2003^{5}$, entendida como una dimensión de la inclusión social, concepto que fue promovido por la Unión Europea (UE) alrededor de la década de 1990, de manera de ampliar la noción de pobreza ya

\footnotetext{
5 Aquí la inclusión digital se define como un conjunto de políticas públicas relacionadas con la construcción, administración, expansión, ofrecimiento de contenidos y desarrollo de capacidades locales en las redes digitales públicas, en cada país y en la región.
} 
que no sólo se refiere a la carencia de recursos sino a un amplio espectro de carencias en cuanto a inserción social y laboral, materialización de derechos sociales y relaciones sociales (Ministerio de Sanidad, Política Social e Igualdad, 2011). En definitiva es un concepto ambiguo y relacional -respecto de la exclusión- que cuenta con al menos tres dimensiones: una discursiva e ideológica, otra institucional o política (procesos de decisión), y una operativa. De manera que depende de la orientación ideológica de que se trate se priorizan diferentes estrategias para las políticas públicas.

En estrecha relación con este concepto es posible entender la inclusión digital como camino de inclusión social. Este concepto entonces, se operacionaliza en función de las dimensiones e indicadores de la brecha digital, y del concepto apropiación tecnológica, donde apropiarse no es solamente uso o consumo, sino apropiarse del objeto tecnológico y de los significados que el objeto transfiere, posibilita o desencadena (Morales, 2009).

Desde hace dos décadas se implementaron innumerables políticas públicas para la reducción de la brecha digital y la inclusión digital en los países latinoamericanos. En Argentina, en la presente década es donde se realizan las más importantes inversiones y políticas para la disminución de la brecha digital que están siendo implementadas por las distintas áreas del Poder Ejecutivo Nacional6. En esta investigación específicamente seleccionamos y abordamos el estudio de la política de alcance nacional el Programa Núcleos de Acceso al Conocimiento (NAC).

\section{Cartografía de políticas de inclusión digital en el Cono Sur de América Latina}

A continuación se describen por país los diversos programas de carácter nacional que se enmarcan dentro de las políticas públicas digitales.

\footnotetext{
${ }^{6}$ Estas son: Sistema Argentino de Televisión Digital Terrestre; Mi TV Digital-Plan de Acceso; Televisión Digital Satelital; Programa de Polos Audiovisuales Digitales; Programa Conectar Igualdad; Agencia Federal para la Sociedad de la Información; Programa Nacional para la Sociedad de la Información (PSI); Programa Núcleos de Acceso al Conocimiento (NAC); Servicio Universal de las Telecomunicaciones; Agenda Digital; Plan Nacional Igualdad Cultural.
} 
En Brasil, cabe destacar el carácter de largo plazo de las políticas de inclusión digital desarrolladas en el país, las cuales presentan continuidad en el tiempo pese a los cambios de gobierno ${ }^{7}$. Las políticas nacionales de inclusión digital son desarrolladas por el Estado de Brasil, que se constituye aquí como actor clave, en conjunto con los estados y municipios que son permanentemente interpelados, además de la participación del sector privado, tanto nacional como internacional, y organismos internacionales. A nivel nacional, encontramos varias políticas destinadas a la inclusión digital de la población brasileña rural y con bajos índices de desarrollo: desde 2003 Casa Brasil, en 2008 Territorios Digitales, en 2011 Inclusión Digital en Juventud Rural. Dirigidas específicamente a la conectividad, en 2005 Goberno ElectrônicoServiço de Atendimento Ao Cidadao (GESAC) ${ }^{8}$. A través del Decreto 7175, en marzo de 2010 se aprueba el Programa Nacional de Banda Larga Brasil Conectado (PNBL). Como parte del PNBL, en 2013 se lleva a cabo el Desoneração de Smartphones. $Y$ en relación al gobierno electrónico, se implementa desde el año 2011 la política Ciudades Digitales para modernizar la gestión, aumentar el servicio a los accesos públicos y proveer el desarrollo de los municipios mediante la tecnología. Por último, desde 2005 el país lleva a cabo una política de reacondicionamiento de los suministros informáticos ${ }^{9}$. Entre las políticas públicas educativas de relevancia actual, encontramos: Programa Nacional de Tecnología Educacional (ex Proinfo); Programa de Banda Ancha en las Escuelas; Programa Um Computador por Aluno (PROUCA).

Por otro lado, en Uruguay encontramos cinco iniciativas entre las políticas públicas digitales: ANTEL integra, Red de Infocentros USI; Centros MEC, que incluye el Plan Nacional de Alfabetización Digital (PNAD); Educantel; Plan Ceibal. Este último se distingue por ser el primer modelo 1:1 implementado en la región y por tanto marca un claro antecedente y referente en la política pública

\footnotetext{
${ }^{7}$ Las modificaciones que pudieran hacerse sobre determinadas políticas públicas se dirigen hacia la profundización de la misma, manteniendo el objetivo inicial y los logros alcanzados hasta ese momento.

${ }^{8}$ En 2013 este programa inició un proceso de expansión: la Secretaría de Comunicaciones publicó el aviso de que triplicaría los puntos de conexión, el número aumentó de 13.379 puntos de presencia a aproximadamente 29.000 .

${ }^{9}$ Este proyecto supone de una red nacional de reutilización de equipo informático de segundo uso, reacondicionado en los Centros de Reacondicionamientos de Computadoras, que brindan a su vez cursos de capacitaciones y actividades de formación
} 
regional en materia de inclusión digital. El actor clave en el diseño, la implementación y el desarrollo de los diferentes programas de inclusión digital es el Estado Nacional de Uruguay, que trabaja en conjunto con la empresa estatal de telecomunicaciones ANTEL.

Cabe destacar, a su vez, el fomento desde estas políticas a la utilización de software de licenciamiento libre, como también un enfoque descentralizador, principalmente en el caso de los Centros MEC, intentando ampliar las diversas políticas a lo largo de todo el territorio.

Sin embargo, podemos observar que el foco principal de las políticas de inclusión digital en Uruguay está puesto principalmente en la educación.

En Chile, en mayo de 2013, se anunció el plan de desarrollo digital para el período de 2013-2020: Agenda Digital ImaginaChile, a través de la cual han quedado definidos cinco ejes estratégicos para el desarrollo digital de los próximos años. En líneas generales, la agenda propone facilitar el acceso a las redes y servicios de la sociedad del conocimiento a todos los chilenos, entregar las capacidades necesarias para desenvolverse en la sociedad del conocimiento, apoyando los procesos formativos, a través de las TIC, desde la escuela hasta la vida laboral, generar un entorno que favorezca el desarrollo y el emprendimiento TIC del país, que fomente la acción combinada de los sectores productivos en materia tecnológica y que proporcione herramientas para potenciar la actividad innovadora, crear las condiciones necesarias para incentivar el desarrollo del ciudadano y las empresas en el mundo digital, y mejorar la productividad y la calidad de vida, a través de los servicios del mundo digital. Guiados por los lineamientos de la agenda nacional, se encuentran los proyectos WiFi ChileGob y la Unidad de Modernización del Estado y Gobierno Digital.

En el plano educativo, el programa Enlaces es una de las iniciativas más destacadas y pioneras. Nace en 1992 con el fin de contribuir al mejoramiento de la calidad de la educación mediante la informática educativa y el desarrollo de una cultura digital $^{10}$. Junto con éste, a partir del 2012 se implementa el programa

\footnotetext{
${ }^{10}$ Este programa trabaja con todos los colegios subvencionados de Chile, entregando estrategias de enseñanza con el uso de tecnología, capacitando profesores, ofreciendo talleres para estudiantes y poniendo a disposición recursos educativos digitales e infraestructura.
} 
Conectividad para la Educación a través del cual todos los establecimientos educativos subvencionados reciben Internet. En tanto TIC en el Aula consiste en la entrega de un computador portátil, un proyector multimedia, un telón y un sistema de amplificación de audio a 13.500 aulas chilenas.

Cabe destacar que tanto Uruguay como Chile son los dos países con mayor penetración de Internet de Latinoamérica ${ }^{11}$.

En cuanto a Perú muchas de las políticas de inclusión digital elaboradas por el Estado cuentan con la participación activa y directa de organismos internacionales, empresas tanto nacionales como multinacionales, y distintas organizaciones de la sociedad civil, no sólo en lo respecta al financiamiento, sino a la implementación y desarrollo de la misma, conjuntamente con el Estado. Podemos apreciar que las políticas de inclusión digital implementadas en el país no poseen continuidad en el tiempo ya sea por falta de financiamiento o por cuestiones políticas ${ }^{12}$.

Una de las principales políticas recae en la "telesalud" tanto en el Registro de nacimientos en línea como en el proyecto de Telemedicina. A su vez, se destacan las políticas de Gobierno electrónico, la implementación del voto electrónico en siete distritos del país y la creación de una Comisión Red Dorsal de Fibra Óptica relativa al desarrollo y despliegue de nuevos backbones de fibra óptica que fomenten la conectividad en las diversas regiones del país.

Entre las políticas públicas de inclusión digital educativa podemos observar:: Proyecto Huascarán; DIGETE; Programa Una Laptop por niño; Programa Tic para Aprender.

Dentro de las iniciativas en Ecuador nos interesa destacar: el plan Nacional de Banda Ancha, plan Nacional de Desarrollo de las Telecomunicaciones, el desarrollo de la Televisión Digital Terrestre y el Plan de acceso Universal y Alistamiento digital. Estos planes se encuentran relacionados con la idea de

11 Antel en el Mundo, "Uruguay con mayor penetración de Internet". Recuperado de: http://www.antel.com.uy/antel/antel-en-el-mundo/uruguay-con-mayor-penetracion-de-internet Última fecha de consulta: 30/08/2015.

12 Tal es el caso de los telecentros rurales INICTEL-UNI que pese a tener diez años de vigencia, se han creado hasta el momento 45 telecentros en todo el país, siendo que en las áreas rurales habitan aproximadamente 7.257.989 de personas según los datos del INIEI. 
inclusión digital educativa, sumado al desarrollo de los Infocentros ${ }^{13}$. Este último es un programa que funciona desde el 2011 y busca reducir los niveles de analfabetismo digital en el país (cercano al 30\%), a través de la utilización de siete unidades móviles o aulas móviles que cuentan con equipos de computación y acceso a Internet, estos se desplazan a lo largo del país brindando talleres gratuitos sobre el uso de redes sociales, correo electrónico, buscadores, entre otros.

Cabe destacar la creación del Fondo para el desarrollo de las Telecomunicaciones (FODETEL), centrado en financiamiento de proyectos de inclusión digital y el subsidio a operadoras privadas para estimular la inversión en materia de telecomunicaciones en zonas rurales y urbano marginales.

Para cerrar esta resumida cartografía, en Argentina el principal encargado de implementar políticas públicas de inclusión digital es sin duda el Estado Nacional, existiendo emprendimientos de carácter provincial que tienen un lugar destacado en la aplicación de este tipo de iniciativas (Ciudad de Buenos Aires, San Luis, Río Negro, Mendoza, Santa Cruz y la Rioja). Dentro de las políticas nacionales iniciadas durante la presidencia de Cristina Fernández de Kirchner, se destaca el Plan Nacional de Telecomunicaciones "Argentina Conectada" que cuenta con una Comisión de Planificación y Coordinación Estratégica encargada de ejecutar y coordinar los programas incluidos: ArSat ${ }^{14}$, Red Federal de Fibra Óptica (REFEFO), Red Social de Conocimiento y vinculación ciudadana (CLIC), Punto de Acceso Digital (PAD), Núcleos de Acceso al Conocimiento (NAC). En el eje Inclusión Digital del documento "Planificación Estratégica Plan Nacional de Telecomunicaciones Argentina Conectada" (s/f), se consigna como objetivo “...contemplar políticas públicas transversales que intensifiquen el acceso al conocimiento a fin de garantizar los derechos consagrados de acceso a la

\footnotetext{
${ }^{13}$ Espacios que buscan acercar a la comunidad a la tecnología y familiarizarla con el uso de las TIC.

${ }^{14}$ Empresa Argentina de Soluciones Satelitales (ArSat): Fundada en 2006 y con sede en Buenos Aires, es una compañía satelital propiedad del Estado argentino que mantiene los derechos exclusivos para operar y comercializar la posición orbital geo estacionaria $81 \mathrm{~W}$ en la banda ku (para América del Norte y del Sur) y en la banda $C$ (para cobertura hemisférica). En ese contexto, la firma lanzó en 2014 su primer satélite geoestacionario, ArSat-1, con el objetivo de fortalecer la cobertura de Internet en el país y la red de DTT, y mejorar la distribución de contenido audiovisual de Argentina para el resto de América Latina y los Estados Unidos.
} 
información y libre expresión, y en definitiva, de posibilitar el ejercicio pleno de la ciudadanía” (p.23).

Asimismo, cabe destacar que en febrero de 2015, el Ministerio de Educación de la Nación Argentina puso en marcha el Plan Nacional de Inclusión Digital Educativa, que integra las diferentes políticas públicas relacionadas con la incorporación de las TIC en las prácticas pedagógicas. Esto incluye al Programa Conectar Igualdad, Primaria Digital y Aulas Rodantes, y se articula con diversas acciones desarrolladas por el Programa "Nuestra Escuela" (de formación docente), entre otras. Al mes de julio de 2015 se han entregado más de 5 millones de netbooks. Estudios de elaboración propia de orden cualitativo y cuantitativo (Lago Martínez y Dughera, 2013; Lago Martínez, 2015) realizados en el Área Metropolitana de Buenos Aires (AMBA), concluyen que el programa posee muchas falencias en su aplicación, relacionadas principalmente con el funcionamiento del piso tecnológico y la capacitación de los docentes.

Cabe destacar que el devenir de muchas de estas políticas públicas de inclusión digital es incierto al no estar completamente definida la forma en que el nuevo gobierno encabezado por el presidente Mauricio Macri planifica su gestión, sus objetivos concretos y su funcionamiento respecto de estas problemáticas en cuestión.

\section{Los Núcleos de Acceso al Conocimiento (NAC) en la problemática}

El Ministerio de Planificación Federal, Inversión Pública y Servicios impulsó el Programa Núcleos de Acceso al Conocimiento (NAC), perteneciente al eje estratégico de inclusión digital del Plan Nacional de Telecomunicaciones Argentina Conectada. Una de dichas metas consiste en el establecimiento de doscientos cincuenta (250) Núcleos de Acceso al Conocimiento (NAC). El propósito del programa es la implementación en todo el territorio nacional de espacios públicos de inclusión digital, ofreciendo a todos los habitantes acceso a la conectividad y a las tecnologías de la Información y Comunicación, para el logro de habilidades digitales, oficios, herramientas de participación, expresión y entretenimiento, de manera gratuita. 
El Estado Nacional asume el compromiso de brindar equipamiento mobiliario y dispositivos tecnológicos, capacitación, contenidos digitales, acompañamiento técnico y administrativo a los NAC, y las instituciones, a aportar el espacio físico y los recursos humanos necesarios para llevar adelante la gestión local de los Núcleos ${ }^{15}$. La mayoría de los NAC se encuentran emplazados en dependencias municipales, entre ellas en Unidades de Gestión Comunitaria (UGC) y en Centros Integradores Comunitarios $(\mathrm{CIC})$ y la minoría en espacios vecinales. Los NAC con sede en la Ciudad de Buenos Aires no están relacionados con el gobierno de la misma sino que responden directamente a la Nación, dos de ellos funcionan en ex centros de detención, tortura y exterminio (Ex Garage Olimpo y Ex ESMA) y otros en centros culturales gestionados por el gobierno nacional, además se incorporan hospitales públicos (como el Hospital de pediatría Garrahan) y Centros de Prevención Local de Adicciones (CePLA) en la ciudad de Córdoba.

En la actualidad (diciembre de 2016), se encuentran instalados 279 NAC en todo el territorio nacional ${ }^{16}$. Cabe mencionar que el que se encuentren "instalados" no implica su funcionamiento al presente. ${ }^{17}$

En el trabajo de campo de nuestra investigación, cuyo periodo de relevamiento abarca desde agosto de 2015 a septiembre de 2016, se relevaron 18 NAC en Capital, GBA, Provincia de Buenos Aires y ciudad de Córdoba. En ellos se realizaron entrevistas a los responsables de los NAC y observaciones de los espacios de funcionamiento. En una segunda etapa se realizaron 250 encuestas al público que asiste a los NAC en diez de los dieciocho Núcleos estudiados. La muestra final alcanzó a 250 casos.

A partir de las entrevistas realizadas a los responsables de cada NAC, de las encuestas a los asistentes y de la observación en cada uno de los espacios físicos, se constató que en general, salvo excepciones, funcionan a lo largo de

\footnotetext{
${ }^{15}$ La oferta de los NAC consiste en: Aprendizaje Tecnológico y Alfabetización digital; Cursos de capacitación; Talleres en temáticas específicas; Formación laboral; Microcine que exhibe la señal emitida por el sistema de Televisión Digital Abierta; Entretenimiento digital; Conectividad inalámbrica; Cursos a través de plataformas virtuales, ya sea en línea (por conexión a internet) y fuera de líneas (por CD interactivo).

${ }^{16}$ Recuperado de: http://www.nac.gob.ar/el-nac-en-cifras, actualmente redirecciona a http://puntodigital.paisdigital.modernizacion.gob.ar/ Ultima revisión: 1/10/2016.

${ }^{17}$ Cabe destacar que al momento de la escritura de la ponencia para el Congreso ALAS (Abril de 2015) el número de NAC era de 198.
} 
todo el día, en amplia franja horaria, algunos se encuentran ubicados en Centros de Integración Comunitarios (CIC) y otros en la sede de municipios o bien en la de organizaciones sociales o civiles.

Casi dos tercios de las personas encuestadas asistentes a los NAC son de sexo femenino (64\%) y las edades predominantes pertenecen a los rangos de 18 a 35 años (30\%) y de 36 a 65 años (32\%) representando el $62 \%$ de los encuestados. Los menores de 18 años y los mayores de 65 asisten en menor medida ( $22 \%$ y $16 \%$ respectivamente).

Respecto a la principal actividad que los usuarios realizan en el NAC, la mayor parte de los concurrentes declara realizar actividades programadas tales como cursos, talleres, capacitación, ver cine, etc. $(57,1 \%)$, mientras que un $26 \%$ declara acercarse sólo a hacer uso de las computadoras. El promedio diario de asistencia es de 2 horas y su mayor frecuencia es de una a dos veces por semana $(77 \%)$.

Respecto a la forma en que se han enterado de la existencia del NAC, la mayoría de la muestra lo ha hecho por el boca en boca $(60,3 \%)$ y en segundo lugar por volantes, afiches y folletos el $16 \%$.

Desde la asistencia a los NAC, el $29 \%$ de los encuestados declaró haber aprendido a usar la computadora e Internet (35\% de las mujeres y 18,5\% de varones y el $77 \%$ de los adultos mayores de 65 años). Cabe destacar que el 53 $\%$ de los usuarios que asisten al NAC declararon ampliar los conocimientos básicos que ya tenían.

En cuanto a la posesión de computadoras en el hogar, el 73,2\% de los usuarios que asisten a los NAC indicaron poseer al menos una computadora de escritorio, portátil o ambas ${ }^{18}$, mientras que un 82,3\% manifestó poseer conexión a Internet de algún tipo. De los usuarios que no tienen computadora y/o Internet, un $87,8 \%$ manifestó utilizar computadoras o conectarse a Internet en el NAC, lo

\footnotetext{
${ }^{18}$ Cabe destacar que el $54 \%$ de los usuarios menores de 18 años poseen solamente computadoras portátiles, este dato está vinculado con los efectos de otros programas de inclusión digital como ser el Conectar Igualdad o el Plan Sarmiento: el 44,5 \% respondió que poseen alguna computadora otorgada por alguno/s de dichos planes.
} 
cual indica cierta efectividad del NAC en el cumplimiento del objetivo de superación de la brecha digital de acceso.

Respecto al conocimiento sobre el programa Núcleos de Acceso al Conocimiento (NAC) llama la atención que tan sólo el $46 \%$ de los asistentes a dicho programa señala que lo conocen, solo el $44 \%$ lo relaciona dentro de la órbita Nacional y sólo el $50,4 \%$ de los encuestados declaró conocer el término "Inclusión Digital" pese a ser los NAC ámbitos diseñados con tal fin.

Pese a esto, el 81,2 \% opinó que los NAC contribuyen a su inclusión digital personal, dentro del cual el $57 \%$ opinó que contribuye mucho.

\subsection{La importancia de los NACs como espacio de inclusión}

En la mirada de los coordinadores de los NAC, los asistentes habituales a estos Núcleos, al hacer uso de las instalaciones y sus equipos, en particular de las computadoras, sienten que se apropian de la tecnología porque tienen su máquina y sus archivos y porque existe una real necesidad de apropiación del conocimiento tecnológico:

\footnotetext{
...de eso es lo que se apropian, de ella, apropiarse de la máquina, servirse, tienen que servirse. Nosotros somos un canal, una ayuda pero ellos tienen que servirse de la tecnología para ser más libres." (Coordinador NAC -Recoleta)

"Yo creo que el que viene al NAC viene porque necesita, porque hay una necesidad de por medio. Porque aquel que no tiene esa necesidad no viene." (Coordinador NAC - Marcos Paz, GBA).
}

Algunos de los coordinadores a cargo de los NAC observan que la brecha digital se va reduciendo en particular en la población más adulta, los mayores van sabiendo cada vez más computación, comienzan a tener su propio correo electrónico y a hacer uso del mismo estableciendo comunicación por este medio. Se conectan a Facebook, crean su propia página de Facebook, aprenden a ver un video, "ingresan al mundo de hoy". Si bien los angustia o les produce temor, lo ven como algo alejado, el poder acceder a un mundo hasta ese momento desconocido. La noción de inclusión digital no es homogénea en todos los entrevistados, en general se entiende como posibilidad de acceso material, físico, a los equipos, se menciona como algo sumamente relevante el tema de la gratuidad, donde está la idea de "desvincular del mercado" a la tecnología. 
También se la asigna importancia al hecho de que el NAC ofrezca un espacio que además contenga a niños, jóvenes y adultos mayores, donde la posibilidad de la recreación (juegos on line y micro cine) cumple un rol importante.

\section{Reflexiones finales}

Entre los países observados para los fines de esta investigación, encontramos varias coincidencias respecto de sus políticas públicas digitales fundamentalmente las orientadas hacia la educación. En primera instancia, en todos los países relevados las políticas tienen como foco prioritario de acción la educación formal básica, ya sea en el nivel secundario o primario. Dentro de este eje de acción podemos distinguir dos líneas principales que orientan dichas políticas: la conectividad de las escuelas a la red de banda ancha y el equipamiento de estudiantes y profesores de computadoras personales, dentro del modelo 1:1. La conectividad en las escuelas está en la mayor parte de los casos asociado a la provisión de computadoras bajo el modelo 1:1, por lo tanto muchos de los programas o planes que se ocupan de la conectividad están enmarcados en estos modelos. No obstante existen otras iniciativas que se ocupan de la conexión a la banda ancha desde programas específicos que funcionan en paralelo a lo relativo específicamente a educación, es decir que piensan la conectividad enfocada a distintas poblaciones prioritarias donde el plano educativo es uno más. Cabe aclarar que los programas tienen varios puntos de divergencia entre sí (respecto del equipamiento, software, modo de distribución, etc.). Los modelos 1:1 son en todos los países observados el centro de las políticas educativas de inclusión digital, esto es debido a su masividad, a la fuerte inversión económica y a su mayor permanencia en el tiempo respecto de otras iniciativas.

Por fuera del sistema formal de educación, existen otras iniciativas que están orientadas a la población que no está alfabetizada digitalmente y que no tiene acceso ya sea a una computadora o a la red de banda ancha en su domicilio. Se trata en la mayor parte de los casos de centros ubicados en diversas partes del país, generalmente en zonas de escasos recursos, que otorgan capacitación a la población y les facilitan el uso de una computadora o de diversos dispositivos digitales dentro de sus instalaciones. 
Otro de los puntos a destacar es que la mayor parte de los países de la región cuenta con una agenda digital a cargo del Estado que prioriza en su plan la inclusión digital educativa. Además podemos observar regularidades en torno a la justificación teórica de estas políticas, todas enmarcadas en el paradigma de la Sociedad de la Información como así también bajo un criterio uniforme de lo que se entiende por inclusión digital. Esto puede resumirse en la idea de que facilitar el acceso y la alfabetización digital de toda la población de un país favorece el ejercicio de derechos ciudadanos en el marco de la Sociedad de la Información.

Siendo el Estado el garante de los derechos colectivos, el mismo es quien debe interceder para la inclusión de los sectores menos favorecidos por la sociedad o aquellos no contemplados por el mercado. De allí la creación de programas como el NAC en Argentina (emprendimientos similares se encuentran en los países de la región) que aspiran a la reducción de la brecha y la inclusión de sectores de la población que no pueden acceder a conectividad, capacitación, formación laboral, etc. Sin embargo, los cambios de signo político principalmente en Argentina, Brasil y Perú abren nuevos interrogantes respecto a la orientación de estas políticas.

Los avances de nuestra investigación respecto a los NAC nos permiten advertir que si bien los concurrentes, en su gran mayoría, ya cuentan con computadoras en el hogar, conexión a Internet y utilizan teléfonos móviles con Internet, buscan incorporar diversas herramientas que les permitan disponer de una mayor formación para el aprovechamiento de las tecnologías digitales. En el caso de los mayores, principalmente aspiran a desarrollar nuevos conocimientos y utilizar las TIC para poder comunicarse, socializar y sentirse incluidos en el mundo digital, mientras que los jóvenes perciben que aprender y conocer sobre tecnologías sirve para capacitarse para su futuro laboral. Observamos también que la oferta de actividades de los NAC es variada, pero se focaliza en las capacitaciones sobre usos básicos e iniciales en el manejo de la computadora e Internet que tienen por objetivo la alfabetización digital, y en menor medida en la formación más específica de herramientas digitales para los que ya cuentan con un cierto conocimiento. 
Respecto a los motivos de asistencia, podemos observar que los usuarios concurren al NAC principalmente para paliar la brecha de conocimiento y en segundo lugar para paliar la brecha tecnológica resultante de no disponer de los dispositivos en su hogar. Otro aspecto destacable, es que desconocen el tipo de software con el que trabajan y la gran mayoría lo hace sólo con software privativo (Microsoft Windows, paquete Office, Adobe), dando cuenta de las limitaciones en cuanto a la apropiación, el reconocimiento de la existencia de alternativas para los usos informáticos, o mismo para la creación de herramientas digitales por parte de los usuarios.

Si bien prácticamente la totalidad de las personas que concurren están satisfechas con el NAC, es interesante destacar que su satisfacción se vincula más con las actividades y el trato recibido que con la posibilidad de desarrollar sus propias iniciativas, ideas y proyectos

A su vez, si bien el programa realiza acciones tendientes a la inclusión digital de los asistentes, no realiza (ni parece tener entre sus planes realizar) acciones en pos de explicar qué significa este término/concepto para lograr una apropiación del mismo (sólo un 50,4\% declaró estar familiarizado con el concepto “inclusión digital” y al consultarle su definición sólo un 14,3\% utilizó el concepto o términos directamente relacionados al mismo). Llama la atención que los niveles socioeconómicos medios sean los que más declaran que contribuye a su inclusión digital dado que se supone que ellos son los que más facilidad y recursos tendrían para suplir por sí mismos las brechas tecnológica y de conocimiento. En relación, podemos indicar que los sectores a los que hipotéticamente más se enfocarían las políticas de inclusión digital son los que menos conocimiento tienen acerca del concepto. En este sentido, el carácter ambiguo del objetivo de "ofrecer a todos los habitantes y en igualdad de condiciones" posibilita esta consecuencia no esperada a priori.

De este modo vemos que el programa NAC se trata mayormente de una política focalizada en los aspectos tecnológicos y económicos y por lo tanto, no termina teniendo en cuenta las dimensiones contextuales y simbólicas de los sujetos que concurren a los NAC. A partir de lo analizado, vemos que el objetivo general del Programa está cumpliéndose, pero que al mismo tiempo ha dejado 
de lado la promoción de una apropiación tecnológica más integral donde los concurrentes se conviertan en partícipes y co-autores de su experiencia en los NAC en lugar de meros usuarios de cursos. Se suma que la mayor parte, como vimos se los capacita utilizando software privativo, lo cual pone en discusión el carácter soberano de la tecnología implementada en la propia gestión de la política pública y en los conocimientos adquiridos que terminan resultando de una forma sesgada en el proceso de aprendizaje de los sujetos.

El devenir de estos espacios públicos ideados originalmente como núcleos para acceder al conocimiento y a la inclusión digital estará signado por la forma en que el gobierno encabezado por el presidente Mauricio Macri decida orientar los objetivos, lineamientos y prácticas de este tipo de políticas públicas, cuestiones que en el transcurso del año de gestión que lleva su gobierno aún no se ha definido. Actualmente se comienza a detectar que los NAC se encuentran en un proceso de descentralización que de la órbita del gobierno nacional pasando a depender su gestión y operatividad de las instituciones huéspedi.

OBS Este trabajo fue presentado como ponencia en el "XXX CONGRESO ALAS Pueblos en movimiento: un nuevo diálogo en las ciencias sociales" realizado del 29 de noviembre al 4 de diciembre del 2015 en San José de Costa Rica. Para este artículo los autores realizaron algunas actualizaciones de los resultados de la investigación sobre el Programa Núcleos de Acceso al Conocimiento en el marco del proyecto "Políticas públicas de Inclusión digital en Argentina y el Cono Sur" que con la dirección de Silvia Lago Martínez se desarrollada en el Instituto Gino Germani, UBA, programación científica 2014-2017.

\section{Refereiincias bibliográficas}

Comisión de Planificación y Coordinación Estratégica (s/f). Documento Planificación Estratégica Plan Nacional de Telecomunicaciones Argentina Conectada. Argentina: Ministerio de Planificación. Recuperado de: http://www.argentinaconectada.gob.ar/arg/258/14000/eje-de-inclusion-digital.html Última fecha de consulta: 30/08/2015.

CROVI Druetta, D. (2004). Sociedad de la información y el conocimiento, entre lo falaz y lo posible. Buenos Aires: La Crujía.

LAGO MARTÍNEZ, S. (2015). Los jóvenes, las tecnologías y la escuela. En Lago Martínez (Coord.) De tecnologías digitales, Internet y educación formal. Retratos de los planes "una computadora, un alumno (271-296). Buenos Aires: Editorial Teseo.

LAGO MARTÍNEZ S.; DUGHERA L. Un acercamiento posible al Programa Conectar (y la) Igualdad en Ciencias Sociales, N 84 (89-95). Buenos Aires: Facultad de Ciencias Sociales UBA, 2013. 
LAGO MARTÍNEZ, S. (2005). La experiencia de los Centros Tecnológicos Comunitarios en Argentina. En O. Islas (Coord.) Internet y la Sociedad de la Información, Tomo I. (265- 290). Quito: Editorial Quipus, CIESPAL.

MATTELART, A. (2002). Historia de la sociedad de la información. Buenos Aires: Paidós.

MINISTERIO DE SANIDAD, Política Social e Igualdad. Estudio comparado sobre Estrategias de inclusión activa en los países de la Unión Europea, Madrid: Centro de publicaciones. Disponível em: $<$ http://www.msssi.gob.es/ssi/familias/nfancia/inclusionSocial/inclusion SocialEspana/informesyEstudios/estrategias.pdf>, 2011

MORALES S. La apropiación de TIC: una perspectiva, en Morales S. y Loyola M. (coord.) Los jóvenes y las TIC. Apropiación y uso en educación. Córdoba: EClUNC, 2009.

VALDERRAMA, C. Sociedad de la información: hegemonía, reduccionismo tecnológico y resistencias, Nómadas № 36 (13-25). Bogotá: IESCO, 2012.

ii OBS Este trabajo fue presentado como ponencia en el "XXX CONGRESO ALAS Pueblos en movimiento: un nuevo diálogo en las ciencias sociales" realizado del 29 de noviembre al 4 de diciembre del 2015 en San José de Costa Rica. Para este artículo los autores realizaron algunas actualizaciones de los resultados de la investigación sobre el Programa Núcleos de Acceso al Conocimiento en el marco del proyecto "Políticas públicas de Inclusión digital en Argentina y el Cono Sur" que con la dirección de Silvia Lago Martínez se desarrollada en el Instituto Gino Germani, UBA, programación científica 2014-2017.

ii Para la escritura de este trabajo se recibieron los aportes de las investigadoras que forman parte del equipo de investigación: Sheila Amado y Ayelén Álvarez y de los graduados y estudiantes de Sociología: Cristina Alonso, Milagros Vidaurre, Ezequiel Fanego, Flavia Samaniego, Cecilia Soto, Fernando Andonegui, Malena Schilseman, Brian Goldman, Vanesa Garrido y Carolina Otharan que realizaron entrevistas y encuestas en los Núcleos de Acceso al Conocimiento (NAC). 